\title{
Design of Stephenson Linkages that Guide a Point Along a Specified Trajectory
}

\author{
Mark M. Plecnik, J. Michael McCarthy \\ Robotics and Automation Laboratory \\ Department of Mechanical and Aerospace Engineering \\ University of California, Irvine \\ Irvine, California 92697
}

\begin{abstract}
This paper presents a design procedure for Stephenson II and Stephenson III six-bar linkages that guide a point along a specified trajectory. The first step is to identify an RR serial chain with a point on its end-link that traces the required trajectory. Eleven configurations of this chain are selected to provide accuracy points for the synthesis of Stephenson II and III function generators that coordinate the two joint angles of the RR chain.

These function generators can be attached to the RR chain four different ways to obtain four kinds of Stephenson path generators. (1) A Stephenson I path generator and (2) a Stephenson II path generator are obtained by attaching a Stephenson III function generator. (3) Another kind of Stephenson II path generator and (4) a Stephenson III path generator are obtained by attaching a Stephenson II function generator. An example of this synthesis procedure finds six-bar leg designs that produce a desired foot trajectory for each of these cases.
\end{abstract}

Keywords:

linkage synthesis, path generator, six-bar linkage

\section{Introduction}

This paper describes the use of Stephenson II and Stephenson III sixbar function generators to control an RR chain so that it generates a de-

Email addresses: mplecnik@uci.edu (Mark M. Plecnik), jmmccart@uci.edu (J. Michael McCarthy)

Preprint submitted to Mechanism and Machine Theory

August 9, 2015

(C) 2015. This manuscript version is made available under the Elsevier user license

http://www.elsevier.com/open-access/userlicense/1.0/ 
sired trajectory. Eleven configurations of the chain defined by points $P_{j}$, $j=0, \ldots, 10$, along the trajectory provide a set of coordinated joint angles that are used as the accuracy points for the design of Stephenson II and III function generators, Plecnik and McCarthy (2015). The ground link of the function generator becomes the first link of the RR chain, thus the resulting linkage design is a scaled kinematic inversion of the function generator.

The Stephenson II and III function generators can each be inverted two different ways for a total of four different path generators. A Stephenson I path generator and a Stephenson II path generator with its trace point connected to a binary floating link are found through inversion of the Stephenson III function generator. A Stephenson II path generator with its trace point connected to its ternary floating link and a Stephenson III path generator are found through inversion of the Stephenson II function generator. Each path generator is shown in Figure 1.

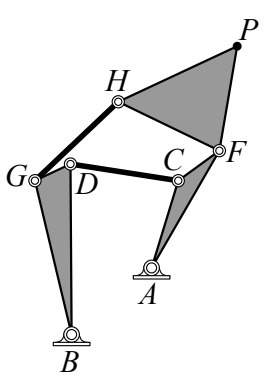

(a)

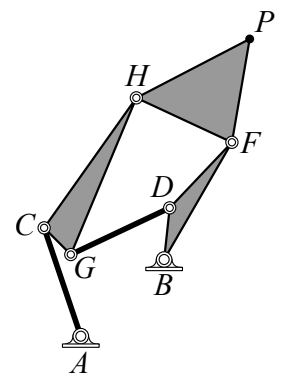

(b)

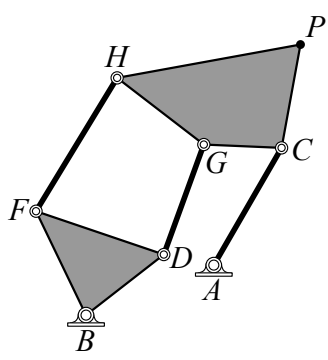

(c)

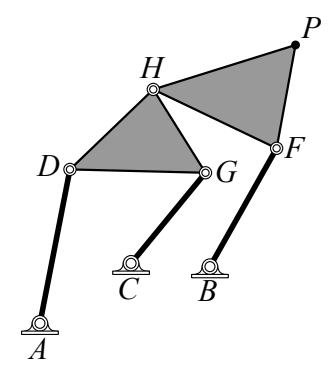

(d)

Figure 1: The four Stephenson six-bar path generators that guide the trace point $P$ : (a) the Stephenson I, (b) the Stephenson II with trace point on a binary floating link, (c) the Stephenson II with trace point on the ternary floating link, and (d) the Stephenson III.

\section{Literature Review}

The synthesis of path generating linkages using a digital computer was pioneered by Freudenstein and Sandor (1959); (1961) who designed four-bar linkages that guide a point through five task points on a desired trajectory. Roth and Freudenstein (1963) presented a numerical solution to the problem of designing a four-bar linkage that reached nine task points on a trajectory, also see Wampler et al. (1992). 
Hain (1967) described graphical methods for point path synthesis using six-bar linkages. The synthesis of six-bar linkages that complete simultaneous path and function generation for five task positions was developed by Mohan Rao et al. (1971). Kim et al. (1972) formulated the design equations for Watt I and Stephenson I, II and III path generators and found solutions using an optimization technique. Prasad and Bagci (1974) applied a Gaussian relaxation technique, and Bhatia and Bagci (1977) applied a linear partition technique for the error minimization of Stephenson linkages. Liu and McPhee (2007) used genetic algorithms to find path generating six-bar linkages, and Cabrera et al. (2011), Peñunuri et al. (2011), and Bulatović and Đorđević (2012) have designed six-bar linkages to generate a path using various differential evolution algorithms.

Our work differs from the previous work in that we use a direct solution of the 11 accuracy point synthesis equations for the six-bar function generator, Plecnik and McCarthy (2015), in order to control the movement of an RR chain. In this way, it similar to the work of Soh and McCarthy (2008) and Plecnik and McCarthy (2013), who constrain open serial chains to create six-bar linkages to reach five task positions. Solutions to the function generation synthesis equations are obtained using the numerical homotopy software BERTINI, see Bates et al. (2013).

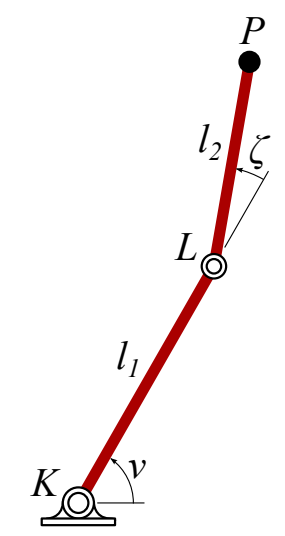

Figure 2: The user defined RR chain with a trace point that defines the required trajectory.

\section{The RR Chain}

The synthesis procedure begins with the specification of an RR chain, see Figure 2. The ground pivot, moving pivot, and trace point are located by 
$K, L$, and $P$, respectively. Note that complex arithmetic is chosen to model planar kinematics in this paper so that,

$$
\begin{aligned}
K & =K_{x}+K_{y} i, \\
L & =L_{x}+L_{y} i, \\
P & =P_{x}+P_{y} i,
\end{aligned}
$$

where the real and imaginary parts represent the horizontal and vertical coordinates of each pivot, respectively. The angle $\nu$ of the first link of the $\mathrm{RR}$ chain is measured from global horizontal. The angle $\zeta$ of the second link is measured relative to the first link. Complex numbers can be rotated in the complex plane by multiplying them by exponential rotation operators. We define

$$
V=e^{i \nu}, \quad Z=e^{i \zeta},
$$

as rotations by $\nu$ and $\zeta$. The location of the trace point $P$ can then be written as

$$
P=K+V l_{1}+V Z l_{2}
$$

Next, we solve for coordinated angle pairs $(\nu, \zeta)$ that move the trace point through a set of specified points $P_{j}$, for $j=0, \ldots, 10$. The angle $\zeta$ is related to the joint coordinates by the the law of cosines, where

$$
(P-K)(\bar{P}-\bar{K})=l_{1}^{2}+l_{2}^{2}-2 l_{1} l_{2} \cos (\pi-\zeta),
$$

where the overbar notation indicates the complex conjugate. Instead of solving for $\zeta$ directly, it is convenient to substitute,

$$
Z+\bar{Z}=-2 \cos (\pi-\zeta)
$$

which is shown to be true using equation (2) and Euler's formula. The terms are rearranged to obtain,

$$
l_{1} l_{2} Z+l_{1}^{2}+l_{2}^{2}-(P-K)(\bar{P}-\bar{K})+l_{1} l_{2} \bar{Z}=0 .
$$

Since $\bar{Z}=\frac{1}{Z}$, equation (6) is quadratic when multiplied by $Z$ and can be solved by the quadratic formula to obtain two solutions,

$$
\begin{aligned}
Z^{ \pm}= & \frac{1}{2 l_{1} l_{2}}\left((P-K)(\bar{P}-\bar{K})-l_{1}^{2}-l_{2}^{2}\right. \\
& \left. \pm \sqrt{\left((P-K)(\bar{P}-\bar{K})-l_{1}^{2}-l_{2}^{2}\right)^{2}-4 l_{1}^{2} l_{2}^{2}}\right)
\end{aligned}
$$


Equation (7) indicates "+" and "-" solutions which correspond to elbow up and elbow down configurations of the RR chain. The corresponding solutions of $V$ are found from solving equation (3),

$$
V^{ \pm}=\frac{P-K}{l_{1}+\left(Z^{ \pm}\right) l_{2}} .
$$

Equations (7) and (8) are computed for $P=P_{j}$, for $j=0, \ldots, 10$, to obtain values $\left(V_{j}, Z_{j}\right)$. For each position, it is the free choice of the designer to select an elbow up or elbow down configuration. The coordinated pairs $\left(V_{j}, Z_{j}\right)$ correspond to angle pairs $\left(\nu_{j}, \zeta_{j}\right)$ for $j=0, \ldots, 10$. Therefore, in order to find mechanisms that move the trace point through $P_{j}$, we must constrain the RR chain such that it achieves coordinated angle pairs $\left(\nu_{j}, \zeta_{j}\right)$ for $j=0, \ldots, 10$.

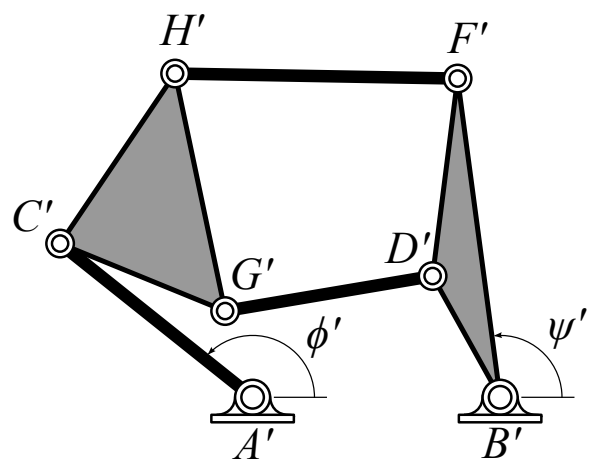

(a)

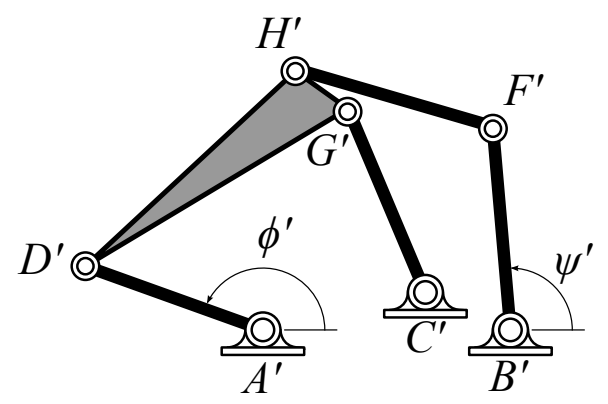

(b)

Figure 3: (a) A Stephenson II function generator and (b) a Stephenson III function generator.

\section{Inversion to Function Generation}

Coordinating the angle pairs $\left(\nu_{j}, \zeta_{j}\right), j=0, \ldots, 10$, is the objective of function generation. We choose to coordinate these angles with six-bar function generators of which only the Stephenson II and III are capable of achieving 11 accuracy points at their ground pivots. Stephenson II and III function generators are shown in Figure 3 where their pivot locations are labelled with primed notation $A^{\prime}, B^{\prime}, C^{\prime}, D^{\prime}, F^{\prime}, G^{\prime}$, and $H^{\prime}$. Furthermore, each of these linkages can be connected to the RR chain (Figure 2) either one of two ways to produce the desired coordination, that is, either joint $A^{\prime}$ lies coincident to $K$ and $B^{\prime}$ lies coincident to $L$ or vice versa, see Figs. 4, 5, 6, and 7. All four resulting types of path generators are described in detail below. 


\subsection{Stephenson I Path Generator}

A Stephenson I path generator is formed by attaching a Stephenson III function generator to the RR chain $K L P$ such that $A^{\prime}$ lies coincident to $K$ and $B^{\prime}$ lies coincident to $L$ as shown in Figure 4 . In this case, ground Link $A^{\prime} B^{\prime} C^{\prime}$ of the function generator is allowed to pivot about $A^{\prime}$ and Link $A^{\prime} D^{\prime}$ is set as the new ground link. The transformation that scales, rotates, and translates the function generator such that $A^{\prime}$ and $B^{\prime}$ line up with $K$ and $L$ is $\mathcal{T}$ defined as

$$
\mathcal{T}(p)=\frac{L-K}{B^{\prime}-A^{\prime}}\left(p-A^{\prime}\right)+K,
$$

where the pivots $A, B, C, D, F, G, H$ of the new path generating linkage are

$$
\begin{aligned}
& A=\mathcal{T}\left(A^{\prime}\right), \quad B=\mathcal{T}\left(D^{\prime}\right), \quad C=\mathcal{T}\left(C^{\prime}\right), \quad D=\mathcal{T}\left(G^{\prime}\right), \\
& F=\mathcal{T}\left(B^{\prime}\right), \quad G=\mathcal{T}\left(H^{\prime}\right), \quad H=\mathcal{T}\left(F^{\prime}\right) .
\end{aligned}
$$

Trace point $P$ is then rigidly attached to Link $F H$ and the Stephenson I path generator is formed. Figure 4 shows the angles $\nu$ and $\zeta$ are related to $\phi^{\prime}$ and $\psi^{\prime}$ of the function generator by

$$
\left(\Delta \phi_{j}^{\prime}, \Delta \psi_{j}^{\prime}\right)=\left(-\Delta \nu_{j}, \Delta \zeta_{j}\right), \quad j=1, \ldots, 10
$$

where

$$
\Delta \phi_{j}^{\prime}=\phi_{j}^{\prime}-\phi_{0}^{\prime}, \quad \Delta \psi_{j}^{\prime}=\psi_{j}^{\prime}-\psi_{0}^{\prime}, \quad \Delta \nu_{j}=\nu_{j}-\nu_{0}, \quad \Delta \zeta_{j}=\zeta_{j}-\zeta_{0} .
$$

Equation (11) sets up the function generation synthesis problem and equation (10) is used to transform the synthesis results of the Stephenson III function generator to Stephenson I path generator results. This procedure is alternative to solving directly for the pivot locations $B, C, D, G, H$ as design parameters of the Stephenson I linkage as a constrained RR chain.

\subsection{Stephenson II Path Generator With Binary Connected Trace Point}

A Stephenson II path generator is formed by attaching a Stephenson III function generator to the RR chain $K L P$ such that $A^{\prime}$ lies coincident to $L$ and $B^{\prime}$ lies coincident to $K$ as shown in Figure 5 . In this case, ground Link $A^{\prime} B^{\prime} C^{\prime}$ of the function generator is allowed to pivot about $B^{\prime}$ and Link $B^{\prime} F^{\prime}$ is set as the new ground link. The transformation that scales, rotates, and 


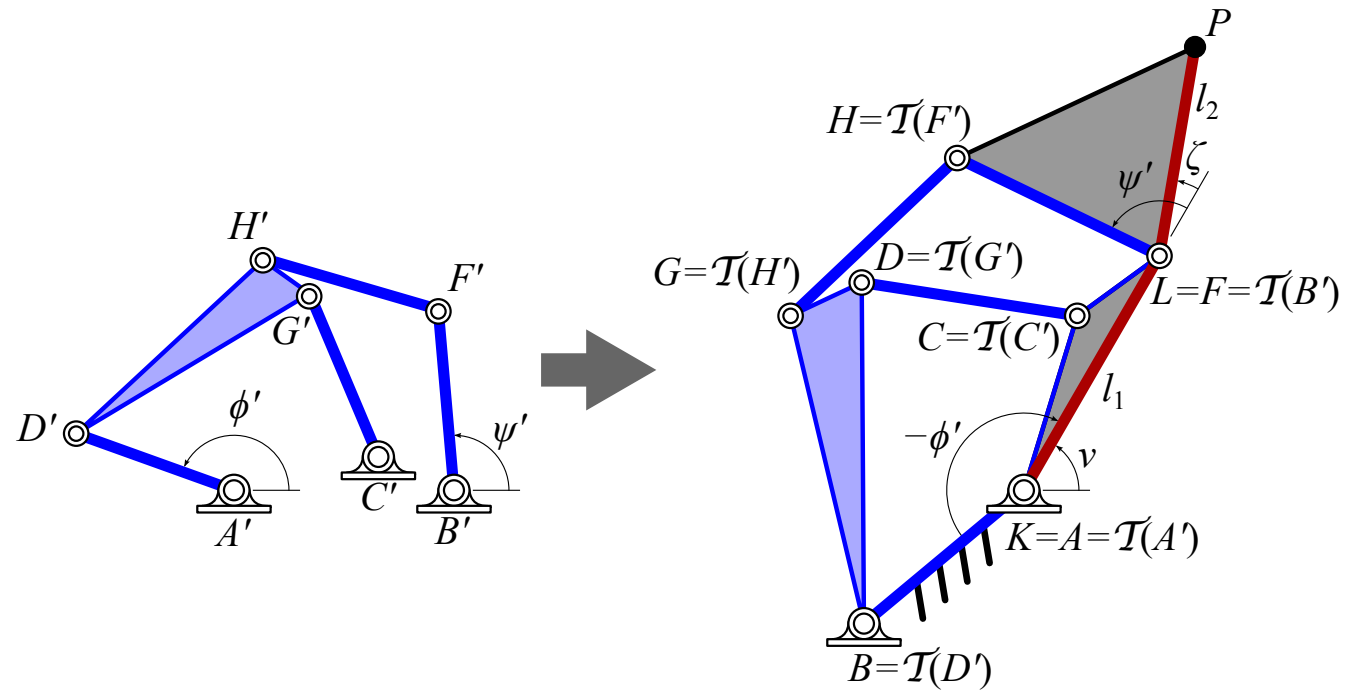

Figure 4: A Stephenson I path generator obtained by coordinating the RR joints with a Stephenson III function generator.

translates the function generator such that $A^{\prime}$ and $B^{\prime}$ line up with $L$ and $K$ is $\mathcal{T}$ defined as

$$
\mathcal{T}(p)=\frac{K-L}{B^{\prime}-A^{\prime}}\left(p-A^{\prime}\right)+L,
$$

where the pivots $A, B, C, D, F, G, H$ of the new path generating linkage are

$$
\begin{aligned}
& A=\mathcal{T}\left(F^{\prime}\right), \quad B=\mathcal{T}\left(B^{\prime}\right), \quad C=\mathcal{T}\left(H^{\prime}\right), \quad D=\mathcal{T}\left(C^{\prime}\right), \\
& F=\mathcal{T}\left(A^{\prime}\right), \quad G=\mathcal{T}\left(G^{\prime}\right), \quad H=\mathcal{T}\left(D^{\prime}\right) \text {. }
\end{aligned}
$$

Trace point $P$ is then rigidly attached to Link $F H$ and the Stephenson II path generator is formed. Figure 5 shows the angles $\nu$ and $\zeta$ are related to $\phi^{\prime}$ and $\psi^{\prime}$ of the function generator by

$$
\left(\Delta \phi_{j}^{\prime}, \Delta \psi_{j}^{\prime}\right)=\left(\Delta \zeta_{j},-\Delta \nu_{j}\right), \quad j=1, \ldots, 10
$$

Equation (15) sets up the function generation synthesis problem and equation (14) is used to transform the synthesis results of the Stephenson III function generator to Stephenson II path generator results. This procedure is alternative to solving directly for the pivot locations $A, C, D, G, H$ as design parameters of the Stephenson II linkage as a constrained RR chain. 


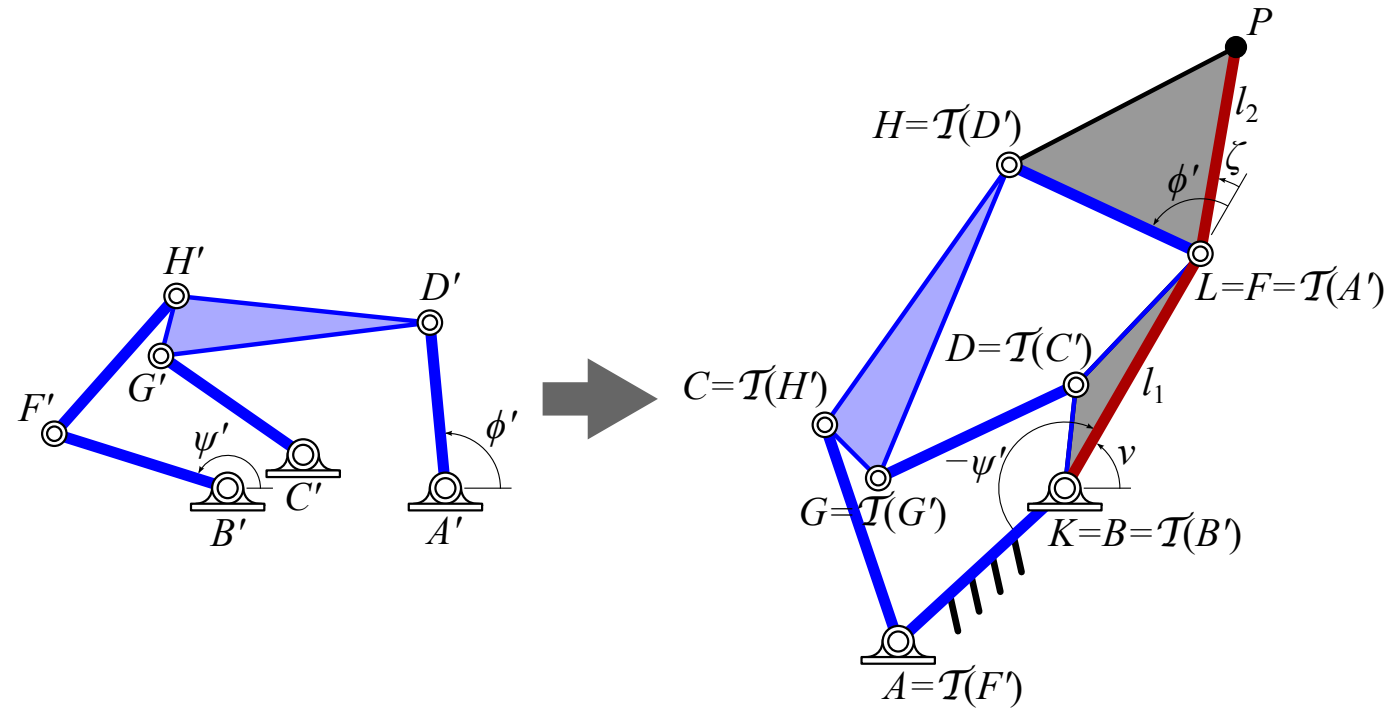

Figure 5: A Stephenson II path generator with trace point on a binary floating link obtained by coordinating the RR joints with a Stephenson III function generator.

\subsection{Stephenson II Path Generator With Ternary Connected Trace Point}

A Stephenson II path generator is formed by attaching a Stephenson II function generator to the RR chain $K L P$ such that $A^{\prime}$ lies coincident to $K$ and $B^{\prime}$ lies coincident to $L$ as shown in Figure 6. In this case, ground Link $A^{\prime} B^{\prime}$ of the function generator is allowed to pivot about $A^{\prime}$ and Link $A^{\prime} C^{\prime}$ is set as the new ground link. The transformation that scales, rotates, and translates the function generator such that $A^{\prime}$ and $B^{\prime}$ line up with $K$ and $L$ is $\mathcal{T}$ defined as

$$
\mathcal{T}(p)=\frac{L-K}{B^{\prime}-A^{\prime}}\left(p-A^{\prime}\right)+K,
$$

where the pivots $A, B, C, D, F, G, H$ of the new path generating linkage are

$$
\begin{array}{lll}
A=\mathcal{T}\left(A^{\prime}\right), & B=\mathcal{T}\left(C^{\prime}\right), & C=\mathcal{T}\left(B^{\prime}\right), \quad D=\mathcal{T}\left(G^{\prime}\right), \\
F=\mathcal{T}\left(H^{\prime}\right), & G=\mathcal{T}\left(D^{\prime}\right), \quad H=\mathcal{T}\left(F^{\prime}\right) .
\end{array}
$$

Trace point $P$ is then rigidly attached to Link $C G H$ and the Stephenson II path generator is formed. Figure 6 shows the angles $\nu$ and $\zeta$ are related to $\phi^{\prime}$ and $\psi^{\prime}$ of the function generator by

$$
\left(\Delta \phi_{j}^{\prime}, \Delta \psi_{j}^{\prime}\right)=\left(-\Delta \nu_{j}, \Delta \zeta_{j}\right), \quad j=1, \ldots, 10
$$


Equation (18) sets up the function generation synthesis problem and equation (17) is used to transform the synthesis results of the Stephenson II function generator to Stephenson II path generator results. This procedure is alternative to solving directly for the pivot locations $B, D, F, G, H$ as design parameters of the Stephenson II linkage as a constrained RR chain.

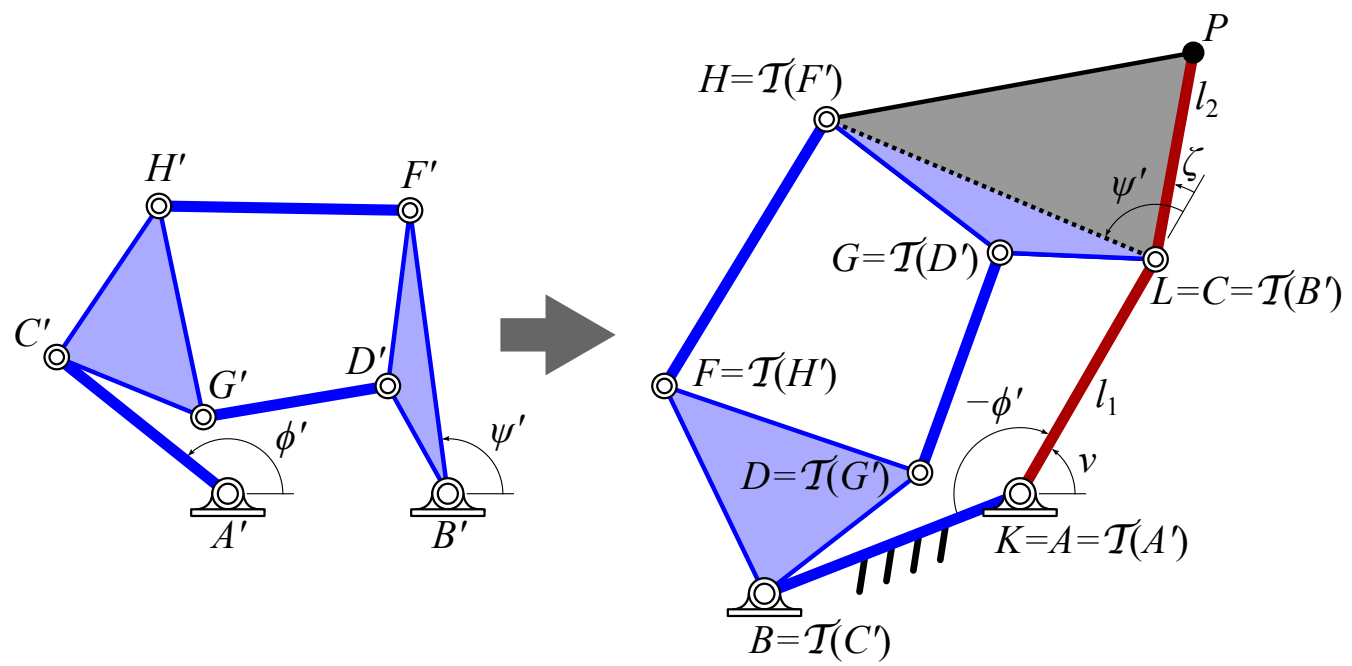

Figure 6: A Stephenson II path generator with trace point on the ternary floating link obtained by coordinating the RR joints with a Stephenson II function generator.

\subsection{Stephenson III Path Generator}

A Stephenson III path generator is formed by attaching a Stephenson II function generator to the RR chain $K L P$ such that $A^{\prime}$ lies coincident to $L$ and $B^{\prime}$ lies coincident to $K$ as shown in Figure 7. In this case, ground Link $A^{\prime} B^{\prime}$ of the function generator is allowed to pivot about $B^{\prime}$ and Link $B^{\prime} D^{\prime} F^{\prime}$ is set as the new ground link. The transformation that scales, rotates, and translates the function generator such that $A^{\prime}$ and $B^{\prime}$ line up with $L$ and $K$ is $\mathcal{T}$ defined as

$$
\mathcal{T}(p)=\frac{K-L}{B^{\prime}-A^{\prime}}\left(p-A^{\prime}\right)+L
$$

where the pivots $A, B, C, D, F, G, H$ of the new path generating linkage are

$$
\begin{aligned}
& A=\mathcal{T}\left(F^{\prime}\right), \quad B=\mathcal{T}\left(B^{\prime}\right), \quad C=\mathcal{T}\left(D^{\prime}\right), \quad D=\mathcal{T}\left(H^{\prime}\right), \\
& F=\mathcal{T}\left(A^{\prime}\right), \quad G=\mathcal{T}\left(G^{\prime}\right), \quad H=\mathcal{T}\left(C^{\prime}\right) .
\end{aligned}
$$


Trace point $P$ is then rigidly attached to Link $F H$ and the Stephenson III path generator is formed. Figure 7 shows the angles $\nu$ and $\zeta$ are related to $\phi^{\prime}$ and $\psi^{\prime}$ of the function generator by

$$
\left(\Delta \phi_{j}^{\prime}, \Delta \psi_{j}^{\prime}\right)=\left(\Delta \zeta_{j},-\Delta \nu_{j}\right), \quad j=1, \ldots, 10 .
$$

Equation (21) sets up the function generation synthesis problem and equation (20) is used to transform the synthesis results of the Stephenson II function generator to Stephenson III path generator results. This procedure is alternative to solving directly for the pivot locations $A, C, D, G, H$ as design parameters of the Stephenson III linkage as a constrained RR chain.

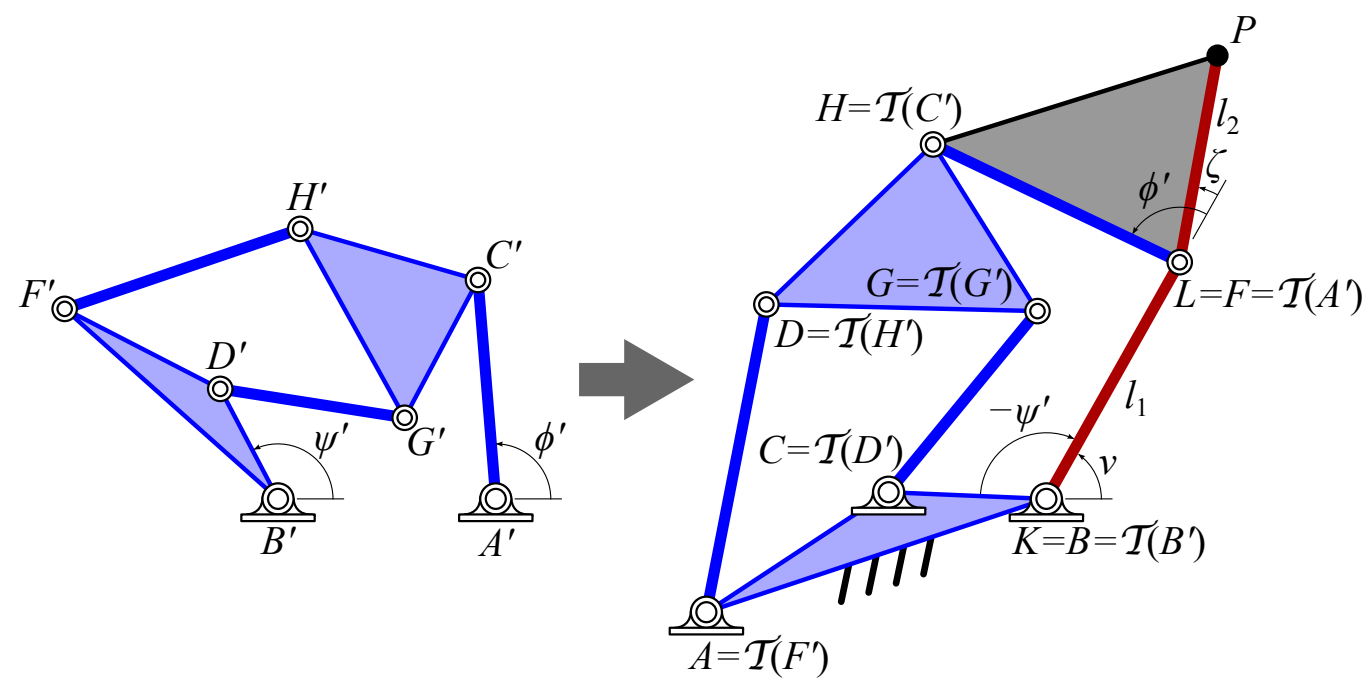

Figure 7: A Stephenson III path generator obtained by coordinating the RR joints with a Stephenson II function generator.

\section{Synthesis of Function Generators}

Section 4 describes four cases in which the problem of path generation is inverted to the problem of function generation. The four cases require solutions for Stephenson II and III function generators that coordinate the angles $\left(\Delta \phi_{j}^{\prime}, \Delta \psi_{j}^{\prime}\right), j=0, \ldots, 10$. The formulation and solution of these synthesis equations is described in Plecnik and McCarthy (2015). For each case, the BERTINI numerical homotopy software was used to find solutions 
to a general form of the synthesis equations resulting in a solution set which can be used to form parameter homotopies which solve in a couple of hours.

Stephenson II and III function generators are displayed in Figure 3. The synthesis equations for both cases take the form,

$$
\begin{array}{r}
\left.\left|\begin{array}{rr}
a \bar{b}_{j} & f \bar{f}-a \bar{a}-b_{j} \bar{b}_{j} \\
c \bar{d}_{j} & g \bar{g}-c \bar{c}-d_{j} \bar{d}_{j}
\end{array}\right| \begin{array}{ll}
\bar{a} b_{j} & f \bar{f}-a \bar{a}-b_{j} \bar{b}_{j} \\
\bar{c} d_{j} & g \bar{g}-c \bar{c}-d_{j} \bar{d}_{j}
\end{array}|+| \begin{array}{cc}
a \bar{b}_{j} & \bar{a} b_{j} \\
c \bar{d}_{j} & \bar{c} d_{j}
\end{array}\right|^{2}=0 \\
j=1, \ldots, 10 .
\end{array}
$$

For the Stephenson II case, joint locations and rotation operators substitute into equation (22) in the following manner,

$$
\begin{aligned}
a & =G^{\prime}-C^{\prime}, & b_{j} & =A^{\prime}-B^{\prime}+Q_{j}^{\prime}\left(C^{\prime}-A^{\prime}\right)-S_{j}^{\prime}\left(D^{\prime}-B^{\prime}\right), \\
c & =H^{\prime}-C^{\prime}, & d_{j} & =A^{\prime}-B^{\prime}+Q_{j}^{\prime}\left(C^{\prime}-A^{\prime}\right)-S_{j}^{\prime}\left(F^{\prime}-B^{\prime}\right), \\
f & =G^{\prime}-D^{\prime}, & g & =H^{\prime}-F^{\prime} .
\end{aligned}
$$

And for the Stephenson III case, the following substitutions into equation (22) are made,

$$
\begin{aligned}
a & =G^{\prime}-D^{\prime}, & b_{j} & =A^{\prime}-C^{\prime}+Q_{j}^{\prime}\left(D^{\prime}-A^{\prime}\right), \\
c & =H^{\prime}-D^{\prime}, & d_{j} & =A^{\prime}-B^{\prime}+Q_{j}^{\prime}\left(D^{\prime}-A^{\prime}\right)-S_{j}^{\prime}\left(F^{\prime}-B^{\prime}\right), \\
f & =G^{\prime}-C^{\prime}, & g & =H^{\prime}-F^{\prime} .
\end{aligned}
$$

Further reductions for each case as well as the use of homotopy to find solutions are discussed in Plecnik and McCarthy (2015).

\section{Performance Verification}

Once function generator solutions are obtained, they are transformed into path generators according to Section 4, forming a set of design candidates. Design candidates must be analyzed to evaluate their performance. The criteria for a successful design is that a path generator reaches all specified points $P_{j}, j=0, \ldots, 10$, on a single trajectory of configurations without passing through a singular configuration. If all points cannot be reached on a single trajectory, then the design has a circuit defect. If the design must pass through a singularity to reach all points, then the design has a branch defect. Singularities are those points where the determinant of the Jacobian of the loop equations is equal to zero. 
Each design candidate was analyzed by formulating its kinematics equations and solving for all configurations when a chosen input parameter was incremented, then sorting those configurations into trajectories. In what follows, we formulate the kinematics equations for Stephenson I, II, and III linkages.

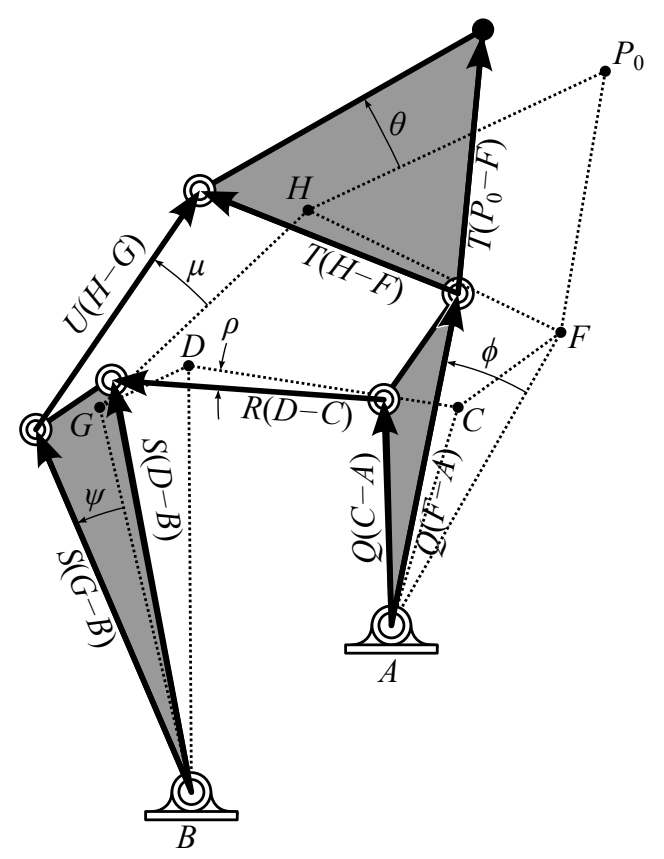

Figure 8: The joint angle conventions for formulation of the loop equations of a Stephenson I six-bar linkage.

\subsection{Stephenson I Kinematics Equations}

A Stephenson I linkage is defined by the locations of its pivots $A, B$, $C, D, F, G, H$, and trace point $P_{0}$ in a reference configuration, see Figure 1(a). This defines the shape of links $A C F, C D, B D G, F H$, and $G H$. When these links are moved from their reference configuration, their orientation is measured by $\phi, \rho, \psi, \theta$, and $\mu$, respectively, see Figure 8 . The exponential rotation operators for each of these angles are

$$
Q=e^{i \phi}, \quad R=e^{i \rho}, \quad S=e^{i \psi}, \quad T=e^{i \theta}, \quad U=e^{i \mu} .
$$


We next form the Stephenson I loop equations,

$$
\begin{aligned}
L & =A-B+Q(C-A)+R(D-C)-S(D-B)=0, \\
M & =A-B+Q(F-A)+T(H-F)-S(G-B)-U(H-G)=0,
\end{aligned}
$$

which can be verified from Figure 8. As well, we form the conjugate loop equations,

$$
\begin{aligned}
\bar{L} & =\bar{A}-\bar{B}+\bar{Q}(\bar{C}-\bar{A})+\bar{R}(\bar{D}-\bar{C})-\bar{S}(\bar{D}-\bar{B})=0 \\
\bar{M} & =\bar{A}-\bar{B}+\bar{Q}(\bar{F}-\bar{A})+\bar{T}(\bar{H}-\bar{F})-\bar{S}(\bar{G}-\bar{B})-\bar{U}(\bar{H}-\bar{G})=0 .
\end{aligned}
$$

In equations (26) and (27), the pivot locations are known from the synthesis results and the joint angle coordinates

$$
\{Q, \bar{Q}, R, \bar{R}, S, \bar{S}, T, \bar{T}, U, \bar{U}\}
$$

remain as unknowns. Furthermore, the joint angle coordinates must have unit magnitude in order to be rotations,

$$
\begin{array}{ll}
N_{Q}=Q \bar{Q}-1=0, & N_{R}=R \bar{R}-1=0, \quad N_{S}=S \bar{S}-1=0, \\
N_{T}=T \bar{T}-1=0, & N_{U}=U \bar{U}-1=0 .
\end{array}
$$

If the angle $\phi$ is chosen as the input, then the input $\mathbf{x}$ and output $\mathbf{y}$ variables are

$$
\mathbf{x}=\{Q, \bar{Q}\}, \quad \mathbf{y}=\{R, \bar{R}, S, \bar{S}, T, \bar{T}, U, \bar{U}\},
$$

and the kinematics equations are

$$
\mathbf{F}(\mathbf{x}, \mathbf{y})=\left\{L, \bar{L}, M, \bar{M}, N_{R}, N_{S}, N_{T}, N_{U}\right\}
$$

This paper analyzes actuation of all links attached to ground for each design candidate. In the case that angle $\psi$ is chosen as the input, equations (30) and (31) change accordingly. For both cases, there are four roots of the kinematics equations $\mathbf{F}(\mathbf{x}, \mathbf{y})$ for a specified input $\mathbf{x}$. Once all joint angle coordinates are found, the location of trace point $P$ is easily calculated.

\subsection{Stephenson II Kinematics Equations}

A Stephenson II linkage is defined by the locations of its pivots $A, B$, $C, D, F, G, H$, and trace point $P_{0}$ in a reference configuration, see Figure 1(c). This defines the shape of links $A C, C G H, B D F, D G$, and $F H$. When 


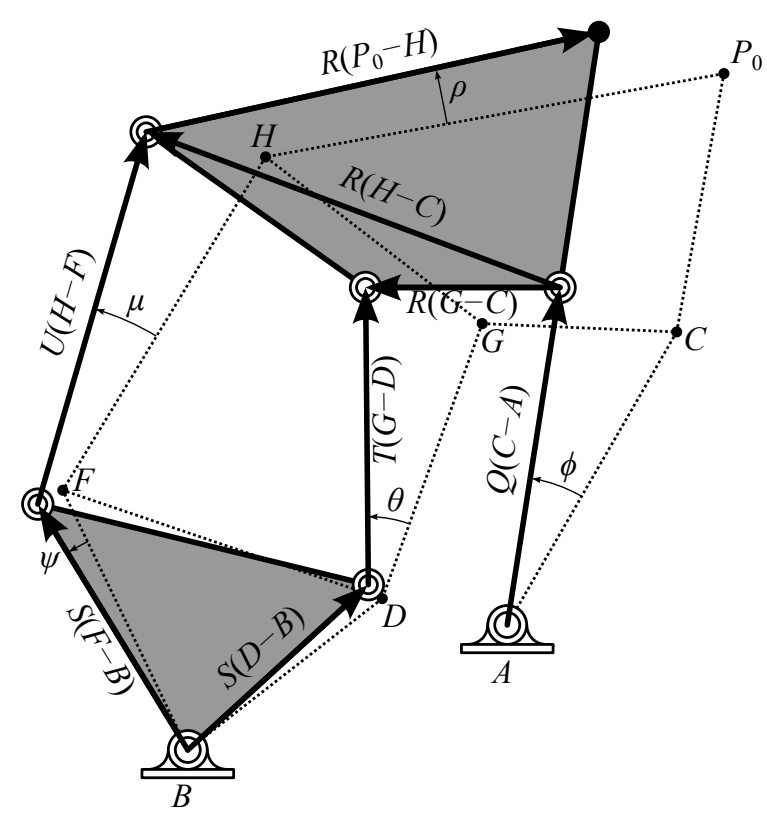

Figure 9: The joint angle conventions for the analysis of the Stephenson II six-bar linkage.

these links are moved from their reference configuration, their orientation is measured by $\phi, \rho, \psi, \theta$, and $\mu$, respectively, see Figure 9 . These angles are represented by the exponential angle operators shown in equation (25). We next form the Stephenson II loop equations,

$$
\begin{gathered}
L=A-B+Q(C-A)+R(G-C)-S(D-B)-T(G-D)=0, \\
M=A-B+Q(C-A)+R(H-C)-S(F-B)-U(H-F)=0,
\end{gathered}
$$

which can be verified from Figure 9. As well, we form the conjugate loop equations,

$$
\begin{gathered}
\bar{L}=\bar{A}-\bar{B}+\bar{Q}(\bar{C}-\bar{A})+\bar{R}(\bar{G}-\bar{C})-\bar{S}(\bar{D}-\bar{B})-\bar{T}(\bar{G}-\bar{D})=0 \\
\bar{M}=\bar{A}-\bar{B}+\bar{Q}(\bar{C}-\bar{A})+\bar{R}(\bar{H}-\bar{C})-\bar{S}(\bar{F}-\bar{B})-\bar{U}(\bar{H}-\bar{F})=0 .
\end{gathered}
$$

In equations (32) and (33), the pivot locations are known from the synthesis results and the joint angle coordinates

$$
\{Q, \bar{Q}, R, \bar{R}, S, \bar{S}, T, \bar{T}, U, \bar{U}\}
$$

remain as unknowns. The joint angle coordinates must have unit magnitude as shown in equation (29). If the angle $\phi$ is chosen as the input, then the 
input $\mathbf{x}$ and output $\mathbf{y}$ variables are

$$
\mathbf{x}=\{Q, \bar{Q}\}, \quad \mathbf{y}=\{R, \bar{R}, S, \bar{S}, T, \bar{T}, U, \bar{U}\},
$$

and the kinematics equations are

$$
\mathbf{F}(\mathbf{x}, \mathbf{y})=\left\{L, \bar{L}, M, \bar{M}, N_{R}, N_{S}, N_{T}, N_{U}\right\} .
$$

This paper analyzes actuation of all links attached to ground for each design candidate. In the case that angle $\psi$ is chosen as the input, (35) and (36) change accordingly. For both cases, there are six roots of the kinematics equations $\mathbf{F}(\mathbf{x}, \mathbf{y})$ for a specified $\mathbf{x}$. Once all joint angle coordinates are found, the location of trace point $P$ is easily calculated.

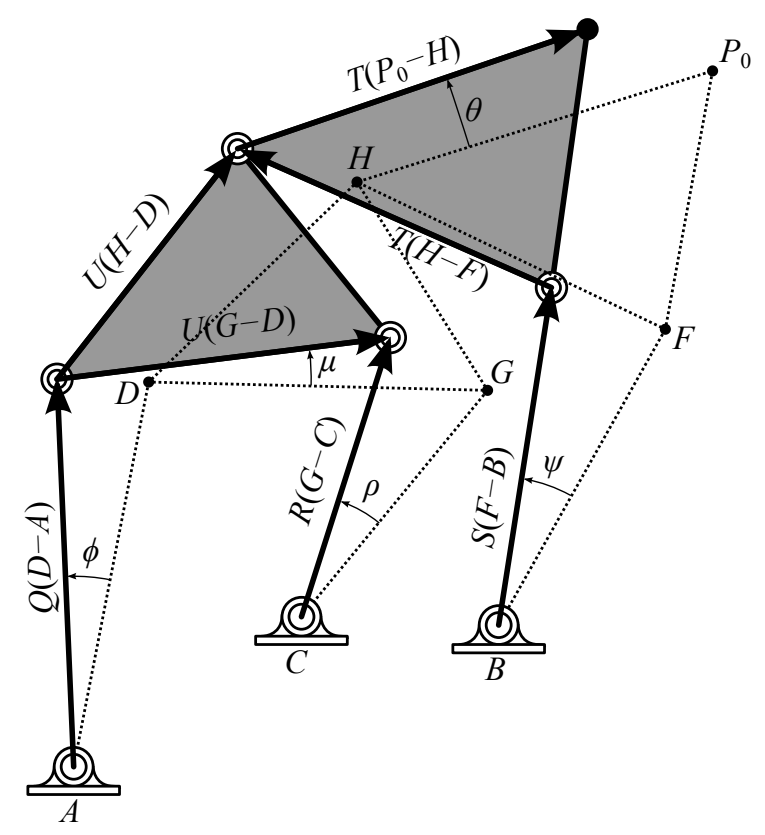

Figure 10: The joint angle conventions for the analysis of a Stephenson III six-bar linkage.

\subsection{Stephenson III Kinematics Equations}

A Stephenson III linkage is defined by the locations of its pivots $A, B$, $C, D, F, G, H$, and trace point $P_{0}$ in a reference configuration, see Figure 1(d). This defines the shape of links $A D, C G, B F, F H$, and $D G H$. When these links are moved from their reference configuration, their orientation is 
measured by $\phi, \rho, \psi, \theta$, and $\mu$, respectively, see Figure 10 . These angles are represented by the exponential angle operators shown in equation (25). We next form the Stephenson III loop equations,

$$
\begin{aligned}
L & =A-C+Q(D-A)+U(G-D)-R(G-C)=0, \\
M & =A-B+Q(D-A)+U(H-D)-S(F-B)-T(H-F)=0,
\end{aligned}
$$

which can be verified from Figure 10. As well, we form the conjugate loop equations,

$$
\begin{aligned}
\bar{L} & =\bar{A}-\bar{C}+\bar{Q}(\bar{D}-\bar{A})+\bar{U}(\bar{G}-\bar{D})-\bar{R}(\bar{G}-\bar{C})=0 \\
\bar{M} & =\bar{A}-\bar{B}+\bar{Q}(\bar{D}-\bar{A})+\bar{U}(\bar{H}-\bar{D})-\bar{S}(\bar{F}-\bar{B})-\bar{T}(\bar{H}-\bar{F})=0 .
\end{aligned}
$$

In equations (37) and (38), the pivot locations are known from the synthesis results and the joint angle coordinates

$$
\{Q, \bar{Q}, R, \bar{R}, S, \bar{S}, T, \bar{T}, U, \bar{U}\}
$$

remain as unknowns. The joint angle coordinates must have unit magnitude as shown in equation (29). If the angle $\phi$ is chosen as the input, then the input $\mathbf{x}$ and output $\mathbf{y}$ variables are

$$
\mathbf{x}=\{Q, \bar{Q}\}, \quad \mathbf{y}=\{R, \bar{R}, S, \bar{S}, T, \bar{T}, U, \bar{U}\},
$$

and the kinematics equations are

$$
\mathbf{F}(\mathbf{x}, \mathbf{y})=\left\{L, \bar{L}, M, \bar{M}, N_{R}, N_{S}, N_{T}, N_{U}\right\} .
$$

This paper analyzes actuation of all links attached to ground for each design candidate. In the cases that either angle $\rho$ or $\psi$ are chosen as the input, (40) and (41) change accordingly. For the cases that either $\phi$ or $\rho$ are the input, there are four solutions to $\mathbf{F}$. If $\psi$ is chosen as the input, there are six solutions to $\mathbf{F}$. Once all joint angle coordinates are found, the location of trace point $P$ is easily calculated.

\section{Sorting Configurations}

The kinematics equations $\mathbf{F}(\mathbf{x}, \mathbf{y})$ of the six-bar linkages in this paper were solved for incremented values of $\mathbf{x}_{k}, k=1, \ldots, n$ such that $\mathbf{x}$ sweeps the unit circle. This was completed for each design candidate. Solutions were found using the NSolve function of MATHEMATICA and then sorted into trajectories following the procedure outlined in Plecnik and McCarthy (2015). 


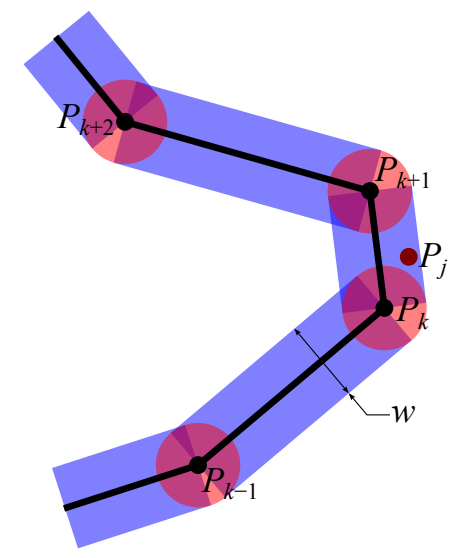

Figure 11: Performance verification requires the linkage candidate trace a trajectory that lies within a small region around the required task points.

\section{Identifying Successful Designs}

Once trajectories have been assembled for a design candidate, each is checked to determine how many of the task points $P_{j}, j=0, \ldots, 10$, it moves through. Trajectories are represented by discrete points generated from a numerical procedure which make it somewhat difficult to determine whether a task point $P_{j}$ belongs to a trajectory $P_{k}, k=1, \ldots, n$. Therefore, each trajectory is "thickened" by constructing a series of circles and boxes at and between each trajectory point $P_{k}$ and $P_{k+1}$ as shown in Fig 11. If $P_{j}$ lies in either a circle or a box, it is said to belong to that trajectory.

The condition for $P_{j}$ to be contained in a circle of diameter $w$ centered at $P_{k}$ is

$$
\left(P_{j}-P_{k}\right)\left(\bar{P}_{j}-\bar{P}_{k}\right)<\frac{w^{2}}{4} .
$$

The conditions for $P_{j}$ to be contained in a box with midline segment defined 
by $P_{k}$ and $P_{k+1}$ and width $w$ are

$$
\begin{aligned}
& 0<\left(P_{j}-P_{k}\right)\left(\bar{P}_{k+1}-\bar{P}_{k}\right)+\left(\bar{P}_{j}-\bar{P}_{k}\right)\left(P_{k+1}-P_{k}\right) \\
&<2\left(P_{k+1}-P_{k}\right)\left(\bar{P}_{k+1}-\bar{P}_{k}\right), \\
&-w \sqrt{\left(P_{k+1}-P_{k}\right)\left(\bar{P}_{k+1}-\bar{P}_{k}\right)} \\
&<i\left(\left(P_{k+1}-P_{k}\right)\left(\bar{P}_{j}-\bar{P}_{k}\right)-\left(\bar{P}_{k+1}-\bar{P}_{k}\right)\left(P_{j}-P_{k}\right)\right) \\
&<w \sqrt{\left(P_{k+1}-P_{k}\right)\left(\bar{P}_{k+1}-\bar{P}_{k}\right) .}
\end{aligned}
$$

Ideally, a design candidate will possess a trajectory that moves through all 11 points, however, we have noticed that design candidates that move through less than 11 points may still have practical value. It is often the case that these mechanisms only slightly miss a task point. Therefore, we keep track of mechanisms that move through 9-11 points.

Table 1: Required task points $P_{j}$ and associated RR joint angles, $\left(\nu_{j}, \zeta_{j}\right)$.

\begin{tabular}{|c|r|r|r|c|}
\hline$j$ & \multicolumn{1}{|c|}{$P_{j}$} & $\nu_{j}(\mathrm{deg})$ & $\zeta_{j}(\mathrm{deg})$ & $\begin{array}{c}\text { Solution } \\
\text { Family }\end{array}$ \\
\hline 0 & $-1.75-4.00 i$ & -97.93 & -28.21 & - \\
\hline 1 & $-1.20-4.00 i$ & -82.11 & -44.03 & - \\
\hline 2 & $-0.60-4.00 i$ & -69.24 & -52.34 & - \\
\hline 3 & $0-4.00 i$ & -59.25 & -54.90 & - \\
\hline 4 & $0.60-4.00 i$ & -52.17 & -52.34 & - \\
\hline 5 & $1.20-4.00 i$ & -48.71 & -44.03 & - \\
\hline 6 & $1.75-4.00 i$ & -50.67 & -28.21 & - \\
\hline 7 & $1.60-3.60 i$ & -98.67 & 58.20 & + \\
\hline 8 & $0.60-3.40 i$ & -125.55 & 80.39 & + \\
\hline 9 & $-0.60-3.40 i$ & -145.56 & 80.39 & + \\
\hline 10 & $-1.60-3.60 i$ & -146.60 & 58.20 & + \\
\hline
\end{tabular}

\section{Example: Leg Mechanism}

The synthesis method described in this paper is illustrated by the design of a leg mechanism for a walking machine that provides a cyclic motion for the foot. The dimensions of the RR chain to be guided were specified as 
Table 2: The number of design candidates and successful designs for the leg mechanism of a walking machine: Stephenson I, Stephenson II (binary floating link), Stephenson II (ternary floating link), and Stephenson III.

\begin{tabular}{|c|c|c|c|c|}
\hline & SI & $\begin{array}{c}\text { SII } \\
\text { (binary) }\end{array}$ & $\begin{array}{c}\text { SII } \\
\text { (ternary) }\end{array}$ & SIII \\
\hline Linkage solutions & 27,000 & 31,469 & 14,358 & 15,545 \\
\hline Design candidates & 14,973 & 17,634 & 5,486 & 4,066 \\
\hline 11-point mechanisms & 36 & 98 & 37 & 4 \\
\hline 10-point mechanisms & 74 & 276 & 54 & 11 \\
\hline 9-point mechanisms & 141 & 469 & 84 & 65 \\
\hline
\end{tabular}

$K=0+0 i, l_{1}=2$, and $l_{2}=2.5$. The task points to trace through and $\mathrm{RR}$ joint angles are displayed in Table 1 . The synthesis algorithm was run for all four Stephenson path generators described in this paper. Statistics on the results for each appear in Table 2. The combined number of 11-point mechanisms was 175, and including 10- and 9-point mechanisms provides 1349 linkage design alternatives.

Example leg mechanisms for each case of the Stephenson path generators are shown in Figure 12; corresponding $(\nu, \zeta)$ functions for each example design appear in Figure 13; and solid models of each example design appear in Figure 14. A solid model of a six-legged alternating tripod gait robot that uses the design shown in Figure 12(b) appears in Figure 15.

\section{Conclusion}

This paper presents a synthesis method for six-bar linkages to trace a required trajectory. It uses the synthesis of a six-bar function generator to control 11 coordinated angles of an RR chain that position a trace point on the required trajectory. The ground link of the function generator becomes the first link of the RR chain and coordinates its two joints. The result is the ability to design linkages that have increased accuracy in tracing curves in the plane. The technique is demonstrated with the design of legs that guide the foot trajectory of a walking machine. The example demonstrates the ability of this technique to generate a large number of design alternatives. 


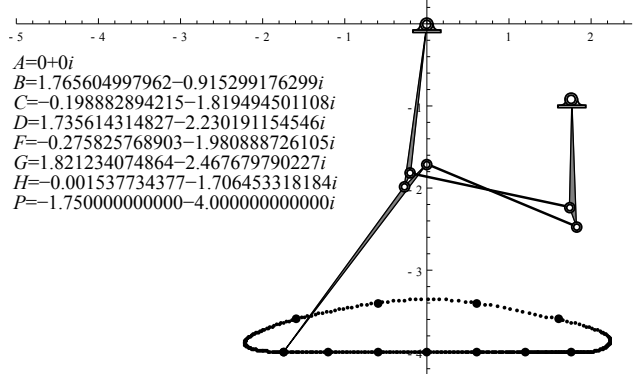

(a)

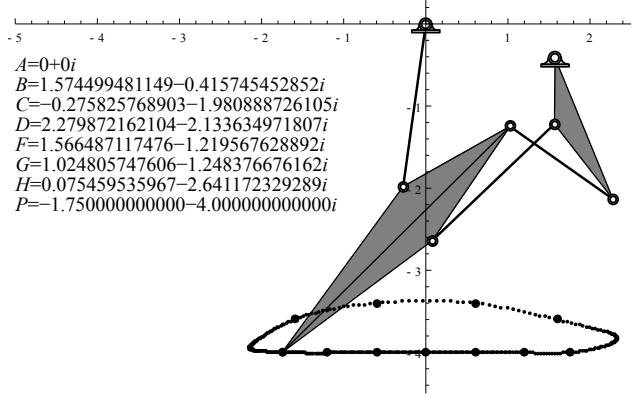

(c)

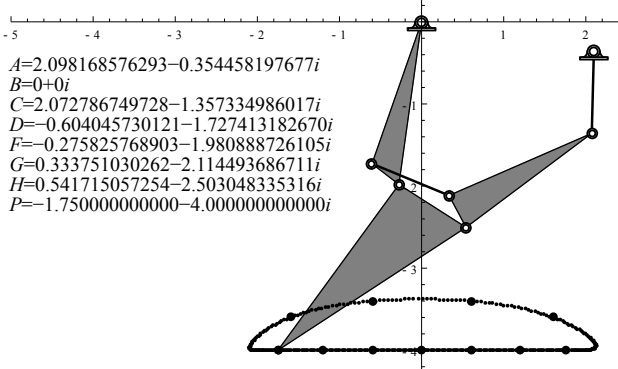

(b)

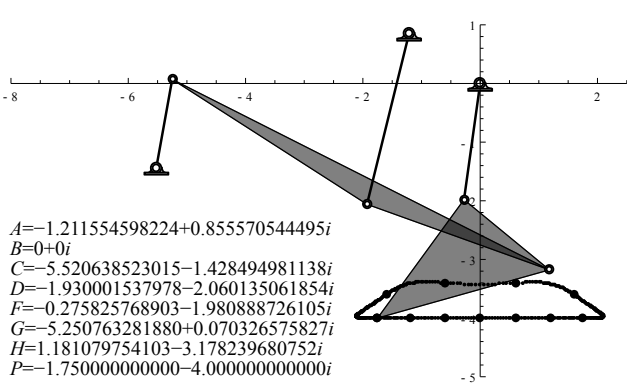

(d)

Figure 12: Successful leg mechanisms for each of the path generators: (a) the Stephenson I, (b) the Stephenson II with trace point on the binary floating link, (c) the Stephenson II with trace point on the ternary link, and (d) the Stephenson III.

\section{Acknowledgments}

This material is based upon work supported by the National Science Foundation under Grant No. CMMI 1066082.

\section{References}

D. J. Bates, J. D. Hauenstein, A. J. Sommese, and C. W. Wampler, 2013. Numerically Solving Polynomial Systems with Bertini, SIAM Press, Philadelphia, PA.

D. J. Bates, J. D. Hauenstein, A. J. Sommese, and C. W. Wampler, 2013. Bertini 1.4: Software for Numerical Algebraic Geometry. Available at bertini.nd.edu with permanent doi: dx.doi.org/10.7274/R0H41PB5. 


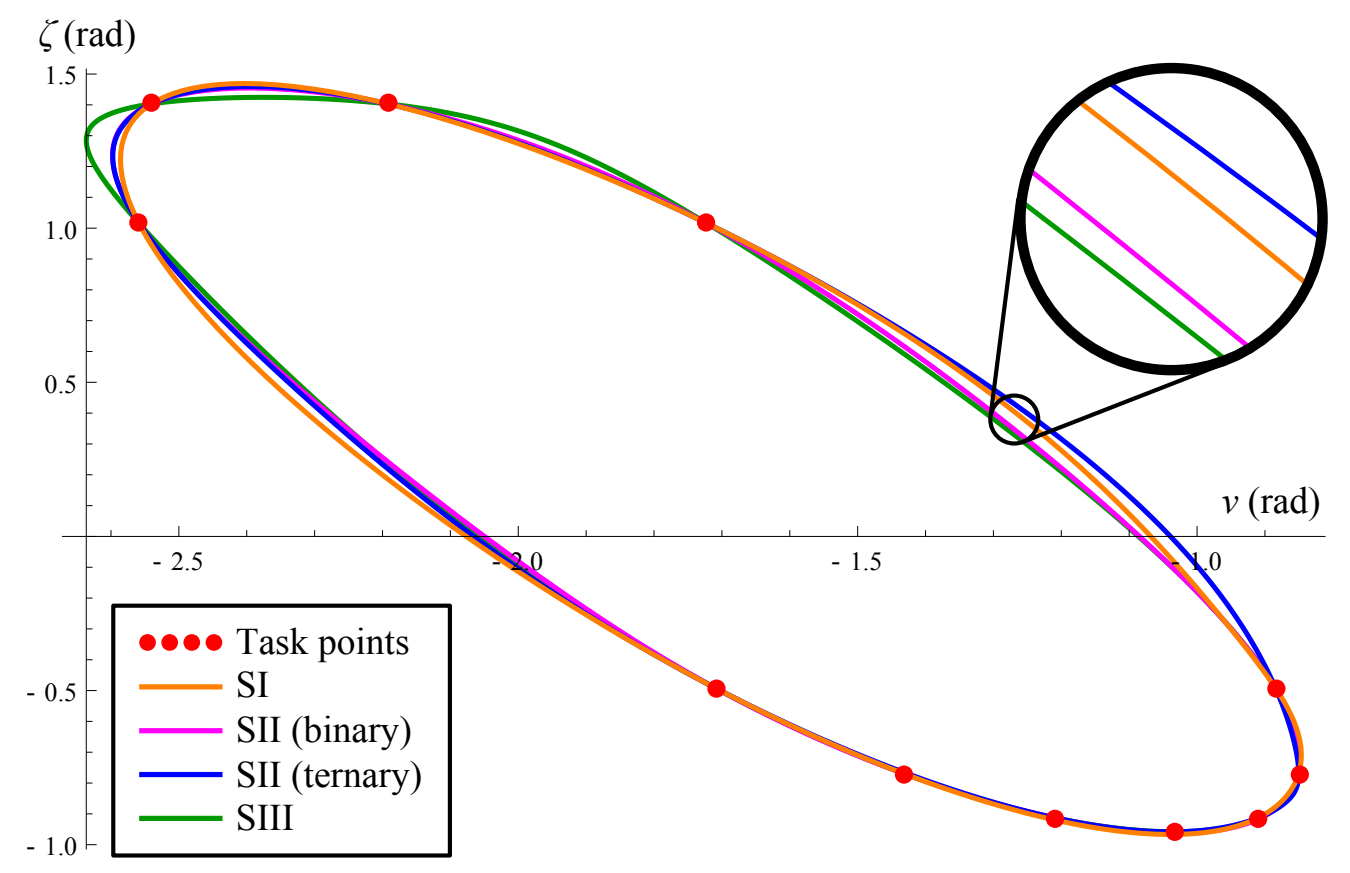

Figure 13: The joint angle trajectories $(\nu, \zeta)$ of the $\mathrm{RR}$ serial chain as controlled by the Stephenson I, Stephenson II (binary floating link), Stephenson II (ternary floating link) and Stephenson III leg mechanisms shown in Figure 12.

D. H. Bhatia and C. Bagci, 1977. Optimum synthesis of multiloop planar mechanisms for the generation of paths and rigid-body positions by the linear partition of design equations, Journal of Engineering for Industry, 99(1):116-123.

R. R. Bulatović and S. R. Đorđević, 2012. "Optimal synthesis of a path generator six-bar linkage," Journal of Mechanical Science and Technology, 26(12):4027-4040.

J. A. Cabrera, A Ortiz, F. Nadal, and J. J. Castillo, 2011. "An evolutionary algorithm for path synthesis of mechanisms," Mechanism and Machine Theory, 46(2):127-141.

F. Freudenstein and G. N. Sandor, 1959. "Synthesis of path generating mechanisms by means of a programmed digital computer," Journal of Engineering for Industry, 81(2):159-168. 


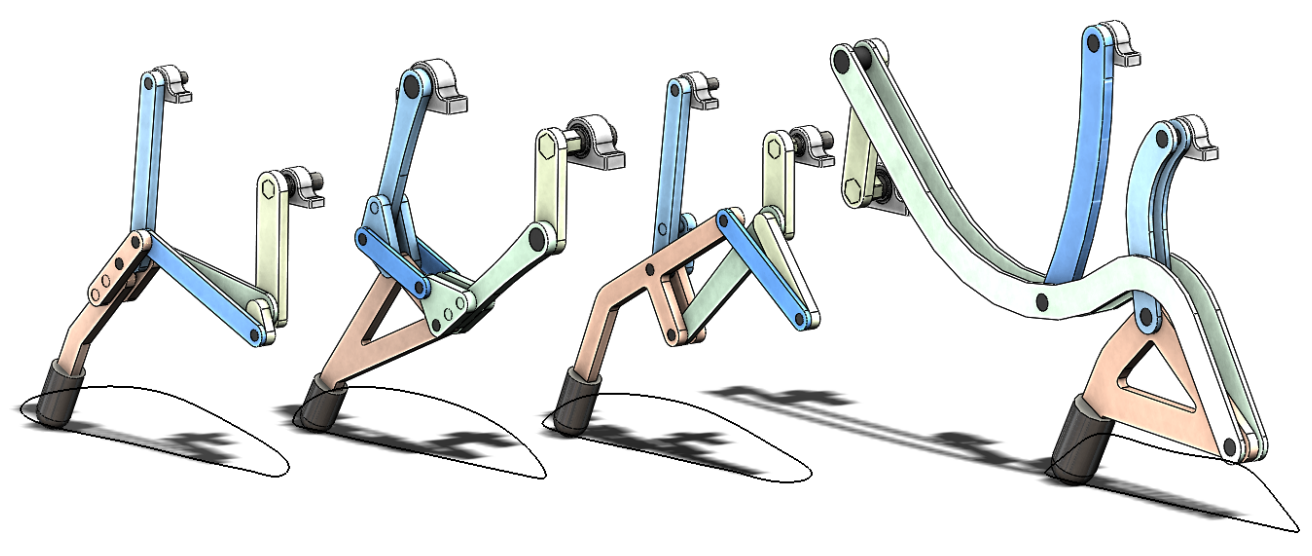

(a)

(b)

(c)

(d)

Figure 14: Solid models of leg mechanisms found from the synthesis results, that is (a) a Stephenson I, (b) a Stephenson II (binary floating link), (c) a Stephenson II (ternary floating link), and (d) a Stephenson III.

F. Freudenstein and G. N. Sandor, 1961. "On the Burmester points of a plane," Journal of Applied Mechanics, 28(1):41-49.

K. Hain, 1967. Applied Kinematics. McGraw-Hill, New York.

H. S. Kim, S. Hamid, and A. H. Soni, 1972. "Synthesis of six-link mechanisms for point path generation," Journal of Mechanisms, 6(4):447-461.

Y. Liu and J. McPhee, 2007. "Automated Kinematic Synthesis of Planar Mechanisms with Revolute Joints," Mechanics Based Design of Structures and Machines, 35(4):405-445.

F. Peñuñuri, R. Peón-Escalante, C. Villanueva, and D. Pech-Oy, 2011. "Synthesis of mechanisms for single and hybrid tasks using differential evolution," Mechanism and Machine Theory, 46(10):1335-1349.

M. Plecnik and J. M. McCarthy, 2013. "Dimensional Synthesis of Six-Bar Linkage as a Constrained RPR Chain," New Trends in Mechanism and Machine Science, Springer Netherlands, 273-280.

M. Plecnik and J. M. McCarthy, 2015. "Computational Design of Stephenson II Six-bar Function Generators for 11 Accuracy Points," accepted for publication in the Journal of Mechanisms and Robotics. 


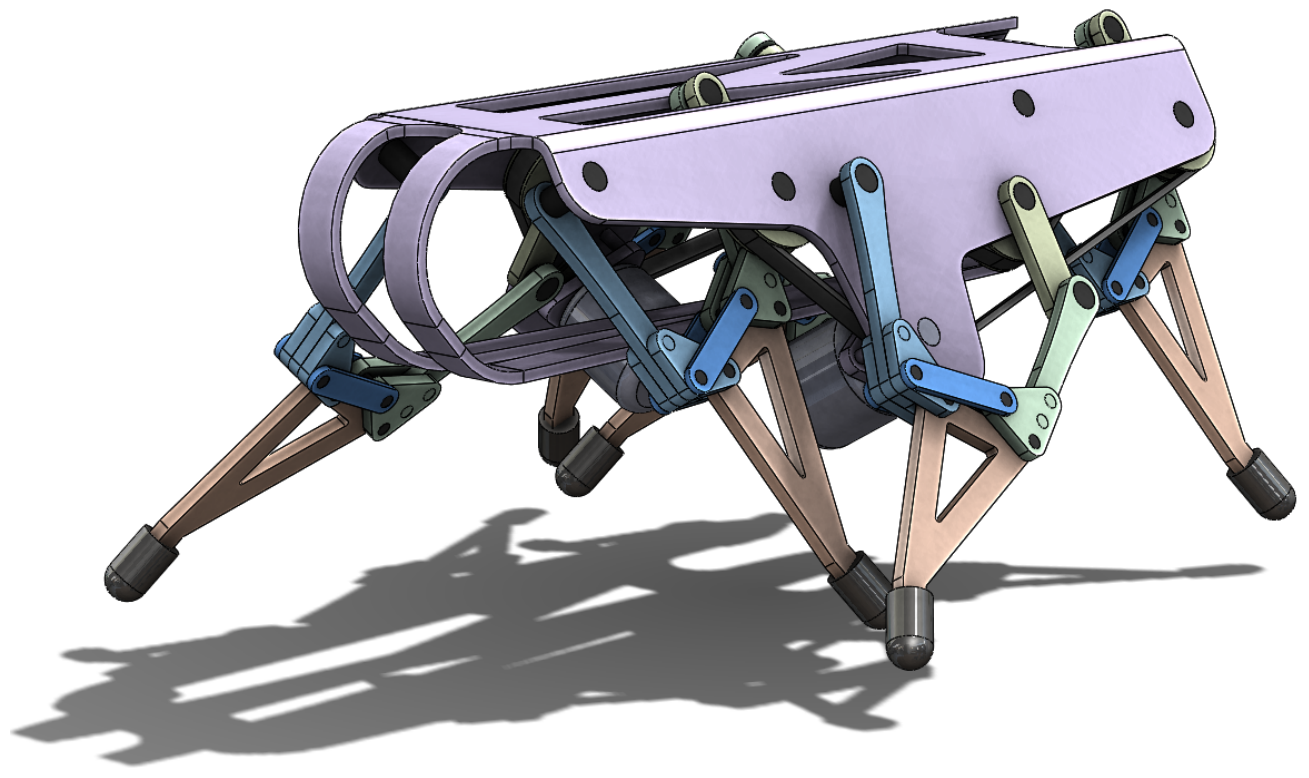

Figure 15: A solid model of a two degree-of-freedom walker, one motor on each side, that uses the Stephenson II leg mechanisms shown in Figure 14(b).

M. Plecnik and J. M. McCarthy, 2015. "Kinematic Synthesis of Stephenson III Six-bar Function Generators," under review for the Mechanism and Machine Theory.

K. N. Prasad and C. Bagci, 1974. "Minimum Error Synthesis of Multiloop Plane Mechanisms for Rigid Body Guidance," Journal of Engineering for Industry, 96(1):107-116.

A. V. Mohan Rao, A. G. Erdman, G. N. Sandor, V. Raghunathan, D. E. Nigbor, L. E. Brown, E. F. Mahardy, and E. D. Enderle, 1971. "Synthesis of multi-loop, dual-purpose planar mechanisms utilizing Burmester theory," Proceedings of the 2nd OSU Applied Mechanism Conference, Paper No. 7, Stillwater, Oklahoma, U.S.A., October 7-8.

B. Roth and F. Freudenstein, 1963. "Synthesis of path-generating mechanisms by numerical methods," Journal of Engineering for Industry, 85(3):298304.

G. S. Soh and J. M. McCarthy, 2008. "The synthesis of six-bar linkages as con- 
strained planar 3R chains," Mechanism and Machine Theory, 43(2):160170.

C. W. Wampler, A. J. Sommese, and A. P. Morgan, 1992. "Complete solution of the nine-point path synthesis problem for four-bar linkages," Journal of Mechanical Design, 114(1):153-159. 
